\title{
Bürgerbeteiligung und die Demokratisierung der Ethik
}

\author{
Claudia Wiesemann
}

Online publiziert: 17 . Oktober 2018

(C) Springer-Verlag GmbH Deutschland, ein Teil von Springer Nature 2018

Eine wichtige Erkenntnis neuzeitlicher Ethik ist, dass ethische Theorien nicht allein deshalb überzeugen, weil sie Ergebnis tiefgründiger Reflektion von Gelehrten sind. Sie müssen sich auf mehr berufen können als nur eine lückenlose, philosophisch stichhaltige Argumentationskette. Sie müssen auch lebensweltliche Erfahrungen widerspiegeln, die Sichtweise der von Entscheidungen betroffenen Personen berücksichtigen und dabei marginalisierten gesellschaftlichen Gruppen eine Stimme verleihen. Ethische Deliberation in pluralen Gesellschaften bedarf der Perspektivenvielfalt, um mehr zu sein als nur ein Herrschaftsinstrument. Sie legitimiert sich auch durch die Art und Weise, wie Betroffene in den Abwägungsprozess eingebunden wurden.

Bürgerbeteiligung gewinnt infolgedessen in der angewandten Ethik mehr und mehr an Bedeutung. Es vergeht kein Tag, an dem in den großen ethischen Debatten der Zeit nicht gefordert wird, die Öffentlichkeit einzubinden. Oft bleibt allerdings unklar, wer „die Öffentlichkeit“" repräsentiert. Bürgerbeteiligungsverfahren werden allenthalben ausprobiert, stecken aber noch in den Kinderschuhen. Was macht - mit Blick auf die bioethischen Fragen der Gegenwart - eigentlich Betroffenheit aus? Ist schon jeder betroffen, der sich diffusen Zukunftsängsten hingibt, oder bedarf es handfesterer Gründe, um einen Anspruch auf Mitsprache zu erwirken (Schicktanz et al. 2008)? Betroffene - oder Stakeholder, wie es auch heißt - können zwar eine normative Position im Diskurs beanspruchen, diese muss aber selbst wiederum einer ethischen Analyse zugänglich sein (Hansen et al. 2018). Worauf gründet sich ihr Anspruch und was besagt er genau? Solche Kontroversen darüber, wer wie und warum gehört werden muss, bereichern die angewandte Ethik, weil sie zugleich

Prof. Dr. C. Wiesemann ( $\square)$

Institut für Ethik und Geschichte der Medizin, Universitätsmedizin Göttingen,

Humboldtallee 36, 37073 Göttingen, Deutschland

E-Mail: cwiesem@gwdg.de 
Auseinandersetzungen darüber sind, welche Erfahrungen und Einstellungen, Werte und Überzeugungen moralisch von Bedeutung sind.

Dass etwa die Beteiligung der betroffenen Patientenverbände bei der Abfassung von Therapieleitlinien nicht nur ein lästiges Zugeständnis an eine empörungsbereite Öffentlichkeit ist, sondern die Diskussion bereichert und wesentlich zur Verbesserung von Empfehlungen beiträgt, spricht sich in der Ärzteschaft langsam herum. Beteiligungs- und Deliberationsverfahren werden allenthalben ausprobiert und werden auch von Ethikgremien als wesentlich für ihre Beratungen verstanden. Der Deutsche Ethikrat etwa veranstaltet bei allen großen Stellungnahmen Anhörungen von Betroffenengruppen. Kaum ein Gremium hat den Gedanken der Bürgerbeteiligung allerdings so innovativ vorangetrieben und derart weit entwickelt wie der britische Nuffield Council on Bioethics, zu dessen Kernaufgaben es gehört, regelmäßig die Öffentlichkeit zu strittigen medizinischen Innovationen zu konsultieren. ${ }^{1}$

In Zukunft wird man jedoch nicht auf den Nuffield Council on Bioethics verweisen, wenn es um die systematische Beteiligung der Öffentlichkeit an Gesetzgebungsprozessen in der Bioethik geht, sondern auf ein Land, das in dieser Hinsicht bisher eher nicht aufgefallen war: Frankreich. Der französische Nationale Ethikrat Comité Consultatif National d'Ethique (CCNE) hat gerade eine Herkulesaufgabe geschultert, die ihresgleichen sucht. Innerhalb von etwas mehr als einem halben Jahr haben sie die Öffentlichkeit befragt, wie sie zu nahezu allen zentralen strittigen Fragen der Bioethik steht, von den Entscheidungen an Lebensanfang bis Lebensende, vom Einsatz künstlicher Intelligenz in der Medizin bis zu Fragen von Umwelt und Gesundheit.

Anlass ist ein unscheinbarer, leicht übersehener Artikel im französischen Bioethikgesetz (Loi relative à la bioéthique) von 2011. ${ }^{2}$ Frankreich regelt seit 1988 alle wesentlichen bioethischen Themen zusammen in einem Gesetz. Bei der letzten Revision des Gesetzes im Jahr 2011 wurde auch der Artikel 46 ergänzt. Er besagt, dass die rechtliche Regelung der Bioethik alle sieben Jahre auf ihre Angemessenheit überprüft werden muss und dass vor einer solchen Revision eine Konsultation der Öffentlichkeit erfolgen muss. Es sollen dazu Bürgerinnen und Bürger zusammenkommen, die die Gesellschaft in ihrer Diversität repräsentieren (,„des conférences de citoyens choisis de manière à représenter la société dans sa diversité"). Als im letzten Jahr dieser Revisionsprozess ins Auge gefasst wurde, versprach die Regierung unter Präsident Emmanuel Macron, der ohnehin in seiner politischen Arbeit schon oft unkonventionelle Formen der Bürgerbeteiligung eingesetzt hat, dieses Vorhaben finanziell zu unterstützen. Seit Anfang 2018 organisierte und koordinierte das CCNE daraufhin Les Etats Généraux de la Bioéthique (die „Generalstände“3 der Bioethik) - wobei mit dem historischen Begriff der Generalstände ein alle Regionen und Bevölkerungsschichten Frankreichs erfassender

\footnotetext{
${ }^{1} \mathrm{Zu}$ den vier Kernaufgaben zählt ein „,appropriate involvement of relevant stakeholders“, http:// nuffieldbioethics.org/about (zugegriffen: 7. Okt. 2018).

${ }^{2}$ LOI n 2011-814 du 7 juillet 2011 relative à la bioéthique, https://www.legifrance.gouv.fr/affichTexte. do?cidTexte=JORFTEXT000024323102 (zugegriffen: 7. Okt. 2018).

3 Ursprünglich waren das Klerus, Adel und sogenannter Dritter Stand.
} 
Beteiligungs- und Konsultationsprozess gemeint ist. Das Motto lautet: „In welcher Welt wollen wir morgen leben?“4

Dazu wurde in allen Regionen Frankreichs mit Hilfe der jeweils dort ansässigen regionalen Ethikkommissionen (ERER) ein Diskussionsprozess initiiert; auch Frankreichs zahlreiche Überseeterritorien wurden einbezogen. Die ERER organisierten 271 Veranstaltungen mit insgesamt 21.000 Teilnehmern. Diskussionsworkshops mit kleiner Teilnehmerzahl zu spezifischen bioethischen Themen fanden oft über mehrere Wochenenden statt. Es wurden neun Themen zur Diskussion gestellt: Entscheidungen am Lebensende, Fortpflanzung und Gesellschaft, künstliche Intelligenz und Robotereinsatz, Umwelt und Gesundheit, Stammzell- und Embryoforschung, genetische Tests und genomische Medizin, Organspende und -transplantation, Neurowissenschaften sowie Umgang mit Gesundheitsdaten. Ein zehntes Thema konnten die Teilnehmerinnen und Teilnehmer des Diskursprozesses selbst einbringen. Sie entschieden sich - nicht ganz überraschend mit Blick auf die immer noch recht paternalistische Medizin in Frankreich - für Fragen der Organisation des Gesundheitswesens, insbesondere der Patientenbeteiligung.

Parallel zu diesen regionalen Diskursveranstaltungen richtete das CCNE eine interaktive Projektwebseite ein, auf der man sich über die oben genannten ethischen Themen informieren und Wünsche und Meinungen äußern konnte. Diese Webseite lockte mehr als 183.000 Besucher an; 29.000 Besucher verfassten insgesamt etwa 65.000 Beiträge. Die überwältigend große Beteiligung überraschte selbst die Organisatoren. Allerdings hatten sie mehrfach Gelegenheit gehabt, im Fernsehen zur Prime Time auf ihr Projekt hinzuweisen. Das CCNE lud auch zu Anhörungen ein. 154 Gruppierungen folgten der Einladung, darunter 88 Patienten- und Selbsthilfegruppen sowie andere Interessengruppen, 36 medizinisch-wissenschaftliche Gesellschaften, neun Gruppierungen mit philosophischem oder religiösem Hintergrund, 18 wissenschaftliche Institutionen (wie das INSERM) und drei Unternehmen.

Der gesamte Prozess wurde durch ein Bürgerkomitee (Comité Citoyen) begleitet. Diese 22 Personen unterschiedlichen Alters, Geschlechts, beruflicher Ausbildung und Wohnorts überwachten den Prozess der Meinungsbildung und gaben selbst einen Bericht zu zwei ethischen Themen ab. Zusätzlich wurde die Position eines Mediators in Konfliktfällen geschaffen. Diese Position wurde mit Louis Schweitzer besetzt, dem ehemaligen Präsidenten der zentralen französischen Institution für Antidiskriminierung und Gleichstellung (Haute autorité de lutte contre les discriminations et pour l'égalité, $H A L D E)$.

Die Auswertung all dieser Konsultationsverfahren wurde unterstützt durch eigens für das Projekt angestellte Mitarbeiterinnen und Mitarbeiter sowie eine professionelle Bürgerbeteiligungsplattform (Cap Collectif ${ }^{5}$ ). Sie verfassten eine detaillierte Darstellung der in den Konsultationen zu den einzelnen Themen vorgebrachten Ar-

\footnotetext{
4 „Quel monde voulons nous pour demain?“ www.etatsgenerauxdelabioethique.fr (zugegriffen: 7. Okt. 2018). Ein Bericht über den gesamten Konsultationsprozess findet sich hier: https://etatsgenerauxdela bioethique.fr/media/default/0001/01/013928888b8655e9c41fac63a51385185d5860c8.pdf (zugegriffen: 7. Okt. 2018).

5 https://cap-collectif.com/ (zugegriffen: 7. Okt. 2018).
} 
gumente, die auf der Webseite abrufbar ist. ${ }^{6}$ Am 18. September 2018 verabschiedeten die Mitglieder des CCNE ihre Stellungnahme zur Revision des Bioethikgesetzes, die mittlerweile der französischen Regierung überreicht wurde. ${ }^{7}$ Darin spricht das CCNE für jedes der oben genannten zehn Themen Empfehlungen aus. Besondere öffentliche Beachtung fand die Empfehlung, die Verfahren der Assistierten Reproduktion auch lesbischen Paaren und alleinstehenden Frauen zugänglich zu machen sowie eine Befruchtung auch nach dem Tod eines der Partner zu ermöglichen. Eine Mehrheit des CCNE sprach sich für eine Aufhebung der Anonymität von Keimzellspendern aus. Aufgrund der hochkontroversen Debatten in den Konsultationen kam das CCNE überdies zu dem Schluss, dass eine Liberalisierung des assistierten Suizids und der Tötung auf Verlangen in Frankreich derzeit nicht ratsam sei.

Noch ist nicht klar, ob das französische Parlament den Empfehlungen des CCNE folgen wird. Aus gutem Grund hat die demokratisch gewählte Nationalversammlung das Recht, sich eine eigene Meinung zu den Themen zu bilden. Aber sie wird nicht leicht am Votum vorbeigehen können, beruht es doch auf einem demokratisch, fair und transparent organisierten nationalen Beteiligungsprozess, der große gesellschaftliche Kräfte mobilisieren konnte. Eine Auswertung des gesamten Prozesses aus gewisser zeitlicher Distanz wird sicherlich noch erforderlich sein. Insbesondere müsste untersucht werden, wie gut die zur Verfügung gestellte Informationsbasis war, ob angemessene Formen der Aktivierung von Diskursteilnehmerinnen und -teilnehmern eingesetzt wurden und gesellschaftliche Randgruppen ausreichend vertreten waren. Auch wäre wichtig, kritisch zu reflektieren, wie Meinungen und Überzeugungen in den philosophischen Argumentationsprozess eingebracht wurden. Aber schon jetzt steht ein wichtiges Ergebnis dieses Kraftaktes fest: Wenn Gesetzgeber in einem parlamentarischen Verfahren moralische Konflikte in gesellschaftlich verträglicher Form rechtlich befrieden müssen, dann sind Bürgerbeteiligungsverfahren ein gangbarer Weg. Sie können den Prozess der ethischen Deliberation auf kreative Weise demokratisieren und somit bereichern.

Interessenkonflikt C. Wiesemann gibt an, dass kein Interessenkonflikt besteht.

\section{Literatur}

Hansen SL, Holetzek T, Heyder C, Wiesemann C (2018) Stakeholder-Beteiligung in der klinischen Forschung: eine ethische Analyse. Ethik Med. https://doi.org/10.1007/s00481-018-0487-7

Schicktanz S, Schweda M, Franzen M (2008) 'In a completely different light'? The role of 'being affected' for the epistemic perspectives and moral attitudes of patients, relatives and lay people. Med Health Care Philos 11:57-72

\footnotetext{
${ }^{6}$ Für die Konsultation über die Webseite dargestellt nach Teilnehmerzahl, Argumenten Pro und Contra sowie besonders zu berücksichtigenden Aspekten: https://etatsgenerauxdelabioethique.fr/media/default/ 0001/01/ae179b8e1a56d82cab2023fd92e52247293943c7.pdf (zugegriffen: 7. Okt. 2018).

7 http://www.ccne-ethique.fr/sites/default/files/avis_129_vf.pdf (zugegriffen: 7. Okt. 2018).
} 